\title{
Compatibility between Chemical \\ Inducers and Amistar Top \\ Fungicide for Controlling Onion \\ Downy Mildew and Purple Blotch \\ Diseases
}

E.Y. Mahmoud; Zeinab N. Hussien;

M.M. Ibrahim and M.A. Abdel-

Gayed

Plant Pathol. Res. Ins., Agric. Res. Center, Giza., Egypt

\begin{abstract}
Three chemical inducers salicylic acid (SA), bion and indol butyric acid (IBA) at different concentrations were applied under field trials to control onion downy mildew and purple blotch and study their effectiveness on onion bulb yield during two successive seasons (2014/2015 and 2015/2016). Chemical inducers were used as a foliar treatment only as well compiled with Amistar Top SC $32.5 \%$ (Azoxstrobin + difenoconazole) as a dipping for onion transplants in permanent field. Recommended fungicide Amistar Top was the best treatment recorded the highest percentage efficacy in reducing downy mildew and purple blotch diseases severity compared to other treatments. Treatment with Chemical inducers showed a significant reduction of downy mildew and purple blotch severity and increased onion bulb yield. In this respect, the highest reduction in disease severity and increase in onion bulb yield were detected with IBA at $200 \mathrm{ppm}$ treatments followed by bion treatment during the two growing seasons. In general, disease severity of downy mildew and purple blotch showed more reduction when onion transplants were dipped in the fungicide before planting. Spraying with IBA at 200 ppm on onion plants treated their transplants by dipping in fungicide tested was the best treatment in reducing downy mildew and purple blotch diseases severity followed by bion treatment at $8 \mathrm{mM}$. Increase the concentration of the chemical inducers caused more reduction in disease severity of both downy mildew and purple blotch diseases. Data clearly indicated that phenolic contents (free, conjugated, and total phenols) and activity of the oxidative enzymes (peroxidase, polyphenol oxidase and catalase) were affected by the three tested concentrations of any chemical inducer. But, applying fungicide as a dipping treatment with different concentrations of chemical inducers had no significant effect on increasing the phenolic contents or the activity of oxidative enzymes in leaves of onion plants during the two growing seasons, 2014-2015 and 2015-2016.
\end{abstract}

Keywords: Allium cepa L, Amistar Top SC 32.5\%, bion, chemical inducers, downy mildew, fungicide, IBA, purple blotch and SA.

Onion (Allium cepa L.) is the most widely cultivated Allium species in Egypt (Mahmoud et al., 2016). Productivity of onion is affected by many biotic and abiotic 
stresses. For commercial cultivation of onion, foliar diseases play the key role in reducing the bulb yield and quality of production. Among the various foliar diseases affecting leaves and bulbs, downy mildew caused by Peronospora destructor (Berk). Caspary and purple blotch caused by Alternaria porri (Ellis) Ciferri are the most devastating and prevalent one (Gupta et al., 2011 and Tripathy et al., 2014). The destructiveness of all these diseases varies widely with locality and season, depending on how often and how long onion foliage is wet by dew (Gupta et al., 2011 and Tripathy et al., 2014).

Downy mildew is one of the major leaf diseases affecting the production of onion bulbs, as well as for onion seeds in the second year which may causes local infection on onion leaves or be systemic and infects the entire plant (Schwartz and Mohan, 2008). Yield reductions of onion bulbs due to downy mildew outbreaks may range from $30 \%$ up to $70 \%$ when the environment is conducive for the disease (Maude, 1990). Yields are strongly reduced by $P$. destructor during seed production, where $60-70 \%$ losses in India may occur (Sharma et al., 2002). Moreover, seeds may be infected by $P$. destructor, acting as primary inoculum for the next season (Rondomanski, 1971). Purple blotch is one of the most devastating diseases, commonly reported in almost all onion growing areas of the world, which causes heavy loss in onions under field conditions (Mustafijur et al., 2015). The disease is however more severe on seed crop as compared to bulb crop causing sometimes $100 \%$ loss of the seed production with average yield loss ranges from 32 to $80 \%$ (Tomaz and Lima, 1988 and Conn and Tewari, 1990).

One of the important management strategies for controlling these diseases is by fungicide applications. Multiple applications of fungicides are usually required to keep onion leaves below damaging levels (Mathur and Sharma, 2006 and Gaikwad et al., 2014). People globally are conscious about environmental deterioration due to use of costly and toxic spray chemicals. So, to save the nature and getting balanced environment, reduce of fungicides usage is needed by finding alternative tools or in some cases reduce of the number of fungicides sprays or their rate (Akter et al., 2015).

Induced disease resistance can be defined as the process of active resistance dependent on the host plants, physical or chemical barriers are activated by biotic or abiotic agents (Meena et al., 2001 and Walters et al., 2007). Some compounds, e.g. ethylene, nicotinic acid, salicylic acid, butyric acid, $\mathrm{K}_{2} \mathrm{HPO}_{4}$ and $\mathrm{KH}_{2} \mathrm{PO}_{4}$ have been shown to induce resistance in plants (Mandal et al., 2009, Hussien, 2011, Ibrahim et al., 2013 and Mahmoud et al., 2014). Induction of systemic resistance sensitizes the plant to respond rapid after infection. These responses include phytoalexin accumulation, phenols, lignifications and activation of many enzymes such as peroxidase, polyphenoloxidase, catalase and chitinase (Meena et al., 2001, Ibrahim et al., 2013 and Mahmoud et al., 2014).

The present study was conducted to investigate the effect of integration between fungicides as dipping treatment and chemical inducers as spraying treatment for reducing onion downy mildew and purple blotch diseases. 


\section{Materials and Methods}

\section{Foliar treatments:}

The effectiveness of three chemical inducers, e.g. salicylic acid (SA) and bion at 2, 4 and $8 \mathrm{mM}$ and indol butyric acid (IBA) at 50; 100 and $200 \mathrm{ppm}$ on disease severity was studied. Each chemical inducer was applied with or without the fungicides dipping treatment of onion transplants. Chemical inducers were used as a foliar spray after 40 and 70 days from transplanting, where the fungicide Amistar top (Azoxstrobin+difenoconazol) was used as a dipping treatment for the transplants at the rate of $75 \mathrm{ml} / 100 \mathrm{~L}$. Meanwhile, Amistar top was also applied as foliar spray, 40 days after transplanting, at the rate of $75 \mathrm{ml} / 100 \mathrm{~L}$. for four times at 15 days intervals.

2. Biochemical changes associated with induced resistance:

Samples of leaves were taken after two days from the second foliar spray and extracted according to Mahmoud et al. (2014), then activities of the oxidative enzymes, i.e. peroxidase (PO); polyphenoloxidase (PPO) and catalase (CAT) were determined according to Allam and Hollis (1972); Matta and Dimond (1963) and Maxwell and Bateman (1967), respectively and assayed using a spectrophotometer at 425,495 and $240 \mathrm{~nm}$., respectively. The reaction substrates of the oxidative enzymes were pyrogallol, catechol and $\mathrm{H}_{2} \mathrm{O}_{2}$ for determining activity of peroxidase; polyphenoloxidase and catalase, respectively. Other samples were extracted in soxhlet units using $75 \%$ ethanol for $10-12$ hrs then used to determine phenol compounds as described by Snell and Snell, (1953), The Phenol contents were also calculated as milligrams equivalent of catechol /g fresh weight of onion leaf, respectively.

3. Field experiments:

Field experiments were carried out during the two successive growing seasons 2014/2015 and 2015/2016 in naturally infested field with Peronospora destructor and Alternaria porri the causal of downy mildew and purple blotch, respectively. The experiment was carried out in Gemiza Experimental St., A.R.C., Gharbia governorate. Randomized complete block design with four replicates was used and the plot was $3.0 \times 3.5 \mathrm{~m}^{2}\left(10.5 \mathrm{~m}^{2}=1 / 400\right.$ feddan $)$. Each plot included 6 rows. Sixty day-old transplants of onion cultivar (Giza 20) were transplanted per each plot at the recommended spacing $10 \mathrm{~cm}$ X $10 \mathrm{~cm}$, within each row on the first week of December, at approximately 75 plants/row. The recommended agricultural practices for onion crop were applied.

\section{Disease and yield assessment:}

a) Disease severity of downy mildew was recorded after three months from transplanting (at first week of March), while purple blotch was recorded after four months from planting (first week of April). One hundred leaves from each plot were chosen as randomized samples to determine disease severity of both studied diseases and was monitored using (0-8) scale and recorded according to the method described by Townsend and Heuberger (1943) as follows:

$0=$ no infection (leaves are completely healthy), 1=1-2 spots per onion leaf, 2 $=3-5$ spots per onion leaf, $3=6-10$ spots per onion leaf, $4=25 \%$ of leaf surface 
was attacked, $5=35-<50 \%$ of leaf surface was attacked, $6=50 \%$ of leaf surface was attacked, $7=75 \%$ of leaf surface was attacked, 8 more than $75 \%$ of leaf surface was attacked. Disease severity index of downy mildew and purple blotch was estimated using the following formula:

$$
\text { D.S.I. }=\frac{\Sigma(\mathrm{n} \times \mathrm{vV})}{\mathrm{ZN}} \times 100
$$

Where:

D.S.I. = Disease severity index, $\mathrm{n}=$ Number of leaves in each category, $\mathrm{v}=$ Numerical value of each category, $\mathrm{Z}=$ Numerical value of highest category and $\mathrm{N}=$ Total number of leaves in the sample.

b) Onion bulb yield as $\mathrm{kg} / \mathrm{plot}\left(10.5 \mathrm{~m}^{2}\right)$ was weighted in all treatments after harvesting.

c) Treatment efficiency (\%) in reducing the disease infection was calculated as

follows:

$\%$ Treatment efficiency $=\{($ Control-treatment $) /$ Control $\}$ X 100

$\%$ Chemical inducer efficiency to fungicide efficacy $($ spray treatment $)=$ $\{$ Ch. Inducer efficiency / fungicide efficacy (spray treatment) $\}$ X 100

\section{Statistical analysis:}

The obtained data were statistically analyzed by analysis of variance (ANOVA) using MSTAT-C program version 2.10 (1991). Means were separation by Duncan test at $\mathrm{P}<0.05$ level.

\section{R e s u l t s}

1. Effect of foliar spraying with some chemical inducers, applied with or without the transplants with the fungicide:

1.1. On downy mildew disease:

Data presented in Tables (1\&2) show that, there was a significant effect of all treatments at their tested concentrations in reducing of downy mildew disease severity during seasons 2014-2015 and 2015-2016 compared to untreated control treatment. In general dipping transplanting of onion in Amistar Top fungicide before planting gave the highest effect in reducing downy mildew disease.

Data in Table (1) also indicate that, IBA treatments followed by bion were the most effective treatments in reducing disease severity and recorded the highest efficacy compared to SA treatments during the two seasons. In this respect IBA at 200 ppm with dipping fungicide application treated was the best treatment in reducing downy mildew disease severity followed by bion at $8 \mathrm{mM}$ under the same conditions during seasons 2014-2015 and 2015-2016 compared to other treatments.

Data also indicate that there was positive correlation ship between increasing the concentration of treatments and their effect on reducing disease severity during the two successive seasons 2014-2015 and 2015-2016. Data in Tables (1\&2) clearly show the ability of some inducers treatments especially with dipping in fungicide treat to be near the fungicide efficiency (spray treatment) in reducing downy mildew disease severity. In this respect, IBA at 200 ppm was the nearest one to fungicide 
efficiency in reducing disease severity of onion downy mildew (93.8\%) during season 2014-2015 while it gave 94.9\% during season 2015-2016.

Table 1. Effect of Amistar Top and some chemical inducers on onion downy mildew severity under field conditions after 3 months from planting during season 2014/2015

\begin{tabular}{|c|c|c|c|c|c|}
\hline \multicolumn{3}{|c|}{ Treatment } & \multicolumn{3}{|c|}{ 2014-2015 } \\
\hline Dipping & Foliar & Conc. & $\begin{array}{l}\text { Disease } \\
\text { severity }\end{array}$ & Efficacy $^{x}$ & $\begin{array}{l}\text { Fungicide } \\
\text { Efficacy }\end{array}$ \\
\hline \multirow{11}{*}{ 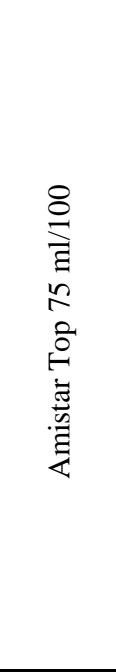 } & SA & $2 \mathrm{mM}$. & $25.94 \mathrm{~d}$ & 56.92 & 65.1 \\
\hline & SA & $4 \mathrm{mM}$. & $21.91 \mathrm{e}$ & 63.62 & 72.8 \\
\hline & SA & $8 \mathrm{mM}$. & $18.15 \mathrm{f}$ & 69.86 & 79.9 \\
\hline & Bion & $2 \mathrm{mM}$. & $21.12 \mathrm{e}$ & 64.93 & 74.3 \\
\hline & Bion & $4 \mathrm{mM}$. & $17.58 \mathrm{gf}$ & 70.81 & 81.0 \\
\hline & Bion & $8 \mathrm{mM}$. & $14.21 \mathrm{ih}$ & 76.40 & 87.4 \\
\hline & IBA & $50 \mathrm{ppm}$ & $16.95 \mathrm{gfh}$ & 71.85 & 82.2 \\
\hline & IBA & $100 \mathrm{ppm}$ & $13.58 \mathrm{i}$ & 77.45 & 88.6 \\
\hline & IBA & 200 ppm & $10.87 \mathrm{j}$ & 81.95 & 93.8 \\
\hline & Amistar Top & $300 \mathrm{ml} / \mathrm{fed}$ & $5.22 \mathrm{k}$ & 91.33 & 104.5 \\
\hline & \multicolumn{2}{|c|}{ Dipping only } & $42.91 \mathrm{~b}$ & 28.74 & 32.9 \\
\hline \multirow{11}{*}{ 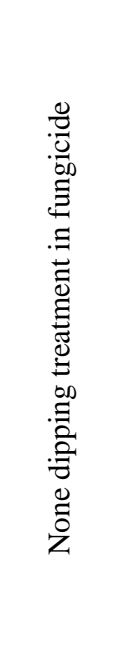 } & SA & $2 \mathrm{mM}$. & $32.22 \mathrm{c}$ & 46.50 & 53.2 \\
\hline & SA & $4 \mathrm{mM}$. & $28.21 \mathrm{~d}$ & 53.16 & 60.8 \\
\hline & SA & $8 \mathrm{mM}$. & $22.87 \mathrm{e}$ & 62.02 & 71.0 \\
\hline & Bion & $2 \mathrm{mM}$. & $25.88 \mathrm{~d}$ & 57.02 & 65.2 \\
\hline & Bion & 4 mM. & $21.88 \mathrm{e}$ & 63.67 & 72.8 \\
\hline & Bion & $8 \mathrm{mM}$. & $17.33 \mathrm{gf}$ & 71.22 & 81.5 \\
\hline & IBA & 50 ppm & $22.12 \mathrm{e}$ & 63.27 & 72.4 \\
\hline & IBA & 100 ppm & 16.22 gifh & 73.07 & 83.6 \\
\hline & IBA & 200 ppm & 14.87 gih & 75.31 & 86.2 \\
\hline & Amistar Top & $300 \mathrm{ml} / \mathrm{fed}$ & $7.58 \mathrm{k}$ & $87.41^{\mathrm{z}}$ & 100.0 \\
\hline & \multicolumn{2}{|c|}{ Control } & $60.22 \mathrm{a}$ & - & - \\
\hline
\end{tabular}

${ }^{\mathbf{x}} \%$ Treatment efficiency $=(($ Control-treatment $) /$ Control $)$ X 100

$\mathbf{y} \%$ Chemical inducer efficiency to fungicide efficacy $\left(\right.$ spray treatment $\left.{ }^{\mathbf{z}}\right)=$

(Ch. Inducer efficiency / fungicide efficacy (spray treatment ${ }^{\mathrm{z}}$ )) X 100 
Table 2. Effect of Amistar Top and some chemical inducers on onion downy mildew severity under field conditions after 3 months from planting during season $2015 / 2016$

\begin{tabular}{|c|c|c|c|c|c|}
\hline \multicolumn{3}{|c|}{ Treatment } & \multicolumn{3}{|c|}{ 2015-2016 } \\
\hline Dipping & Foliar & Conc. & $\begin{array}{l}\text { Disease } \\
\text { severity }\end{array}$ & Efficacy $^{x}$ & $\begin{array}{l}\text { Fungicide } \\
\text { Efficacy }^{\mathrm{y}}\end{array}$ \\
\hline \multirow{11}{*}{ 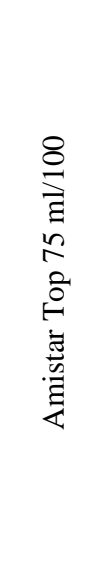 } & SA & $2 \mathrm{mM}$. & $21.53 \mathrm{de}$ & 59.29 & 67.8 \\
\hline & SA & $4 \mathrm{mM}$. & $19.32 \mathrm{ef}$ & 63.47 & 72.6 \\
\hline & SA & $8 \mathrm{mM}$. & $15.35 \mathrm{hg}$ & 70.98 & 81.2 \\
\hline & Bion & $2 \mathrm{mM}$. & $18.09 \mathrm{gf}$ & 65.80 & 75.2 \\
\hline & Bion & $4 \mathrm{mM}$. & $15.22 \mathrm{hg}$ & 71.22 & 81.4 \\
\hline & Bion & $8 \mathrm{mM}$. & $12.55 \mathrm{hi}$ & 76.27 & 87.2 \\
\hline & IBA & $50 \mathrm{ppm}$ & $14.69 \mathrm{~h}$ & 72.23 & 82.6 \\
\hline & IBA & $100 \mathrm{ppm}$ & $11.22 \mathrm{ji}$ & 78.79 & 90.1 \\
\hline & IBA & $200 \mathrm{ppm}$ & $8.99 \mathrm{jk}$ & 83.00 & 94.9 \\
\hline & Amistar Top & $300 \mathrm{ml} / \mathrm{fed}$. & 4.121 & 92.21 & 105.4 \\
\hline & \multicolumn{2}{|c|}{ Dipping only } & $33.95 \mathrm{~b}$ & 35.81 & 40.9 \\
\hline \multirow{11}{*}{ 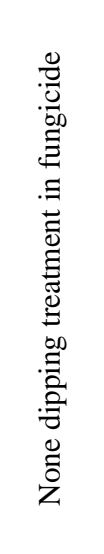 } & SA & $2 \mathrm{mM}$. & $26.53 \mathrm{c}$ & 49.84 & 57.0 \\
\hline & SA & $4 \mathrm{mM}$. & $22.90 \mathrm{~d}$ & 56.70 & 64.8 \\
\hline & SA & $8 \mathrm{mM}$. & $18.32 \mathrm{f}$ & 65.36 & 74.7 \\
\hline & Bion & $2 \mathrm{mM}$. & $23.77 \mathrm{dc}$ & 55.06 & 63.0 \\
\hline & Bion & $4 \mathrm{mM}$. & $20.80 \mathrm{def}$ & 60.67 & 69.4 \\
\hline & Bion & $8 \mathrm{mM}$. & $14.23 \mathrm{hi}$ & 73.10 & 83.6 \\
\hline & IBA & $50 \mathrm{ppm}$ & 19.85 ef & 62.47 & 71.4 \\
\hline & IBA & 100 ppm & $14.58 \mathrm{~h}$ & 72.43 & 82.8 \\
\hline & IBA & $200 \mathrm{ppm}$ & $12.33 \mathrm{hi}$ & 76.69 & 87.7 \\
\hline & Amistar Top & $300 \mathrm{ml} / \mathrm{fed}$. & $6.63 \mathrm{lk}$ & $87.46^{z}$ & 100.0 \\
\hline & \multicolumn{2}{|c|}{ Control } & $52.89 \mathrm{a}$ & - & - \\
\hline
\end{tabular}

$\mathrm{x} \%$ Treatment efficiency $=(($ Control-treatment $) /$ Control $) \mathrm{X} 100$

${ }^{\mathrm{y}} \%$ Chemical inducer efficiency to fungicide efficacy $\left(\right.$ spray treatment $\left.{ }^{\mathrm{z}}\right)=$ (Ch. Inducer efficiency / fungicide efficacy (spray treatment z)) X 100

\subsection{On purple blotch disease:}

Data illustrated in Tables (3\&4) indicate that disease severity of purple blotch was significantly decreased by different treatments of chemicals induce resistance in onion plants and recommended fungicide Amistar Top during two successive seasons compared to untreated control treatment. In general, disease severity of purple blotch was highly affected when onion transplants dipped in fungicide before planting. Data in Tables (3\&4) also, show that recommended fungicide Amistar Top 
was the best treatment recorded the highest percentage efficacy in reducing purple blotch disease severity compared to other treatments.

On the other hand, IBA treatments followed by bion were the most effective treatments in reducing disease severity and recorded the highest efficacy compared to other SA treatments during the two growing seasons 2014-2015 and 2015-2016. In this respect, spraying plants dipped their transplants in the fungicide tested with IBA at $200 \mathrm{ppm}$ was the best treatment in reducing purple blotch disease severity followed by bion at $8 \mathrm{mM}$.

Table 3. Effect of fungicide Amistar Top and some chemical inducers on onion purple blotch disease severity under field conditions during season 2014/2015

\begin{tabular}{|c|c|c|c|c|c|}
\hline \multicolumn{3}{|c|}{ Treatments } & \multicolumn{3}{|c|}{ 2014-2015 } \\
\hline Dipping & Foliar & Conc. & $\begin{array}{l}\text { Disease } \\
\text { severity }\end{array}$ & Efficacy $^{x}$ & $\begin{array}{l}\text { Fungicide } \\
\text { Efficacy }^{\mathrm{y}}\end{array}$ \\
\hline \multirow{11}{*}{ 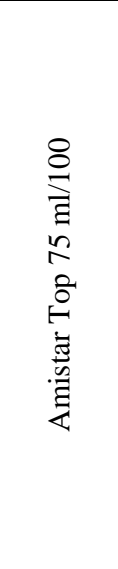 } & SA & $2 \mathrm{mM}$. & $12.01 \mathrm{fe}$ & 70.60 & 79.0 \\
\hline & SA & $4 \mathrm{mM}$. & $9.23 \mathrm{hg}$ & 77.41 & 86.6 \\
\hline & SA & $8 \mathrm{mM}$. & $6.45 \mathrm{kji}$ & 84.21 & 94.2 \\
\hline & Bion & $2 \mathrm{mM}$ & $9.89 \mathrm{fg}$ & 75.79 & 84.8 \\
\hline & Bion & $4 \mathrm{mM}$. & $7.58 \mathrm{hji}$ & 81.44 & 91.1 \\
\hline & Bion & $8 \mathrm{mM}$. & $6.22 \mathrm{kji}$ & 84.77 & 94.9 \\
\hline & IBA & $50 \mathrm{ppm}$ & $7.22 \mathrm{hji}$ & 82.33 & 92.1 \\
\hline & IBA & $100 \mathrm{ppm}$ & $5.40 \mathrm{kj}$ & 86.78 & 97.1 \\
\hline & IBA & $200 \mathrm{ppm}$ & $3.12 \mathrm{ml}$ & 92.36 & 103.4 \\
\hline & Amistar Top & $300 \mathrm{ml} / \mathrm{fed}$ & $1.89 \mathrm{~m}$ & 95.37 & 106.7 \\
\hline & \multicolumn{2}{|c|}{ Dipping only } & $36.85 \mathrm{~b}$ & 9.79 & 11.0 \\
\hline \multirow{11}{*}{ 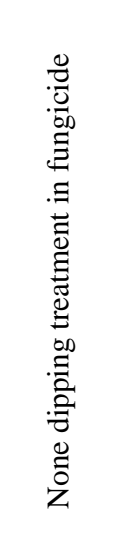 } & SA & $2 \mathrm{mM}$. & $18.7 \mathrm{c}$ & 54.22 & 60.7 \\
\hline & SA & $4 \mathrm{mM}$ & $15.98 \mathrm{~d}$ & 60.88 & 68.1 \\
\hline & SA & $8 \mathrm{mM}$. & $13.41 \mathrm{e}$ & 67.17 & 75.2 \\
\hline & Bion & $2 \mathrm{mM}$. & $16.07 \mathrm{~d}$ & 60.66 & 67.9 \\
\hline & Bion & $4 \mathrm{mM}$. & $12.88 \mathrm{e}$ & 68.47 & 76.6 \\
\hline & Bion & $8 \mathrm{mM}$. & $10.12 \mathrm{fg}$ & 75.23 & 84.2 \\
\hline & IBA & $50 \mathrm{ppm}$ & $13.52 \mathrm{e}$ & 66.90 & 74.9 \\
\hline & IBA & $100 \mathrm{ppm}$ & $10.6 f \mathrm{~g}$ & 74.05 & 82.9 \\
\hline & IBA & $200 \mathrm{ppm}$ & 8.42 hgi & 79.39 & 88.9 \\
\hline & Amistar Top & $300 \mathrm{ml} / \mathrm{fed}$ & $4.35 \mathrm{kl}$ & $89.35^{\mathrm{z}}$ & 100.0 \\
\hline & \multicolumn{2}{|c|}{ Control } & $40.85 \mathrm{a}$ & - & - \\
\hline
\end{tabular}

${ }^{x} \%$ Treatment efficiency $=(($ Control-treatment $) /$ Control $)$ X 100

y $\%$ Chemical inducer efficiency to fungicide efficacy $\left(\right.$ spray treatment $\left.{ }^{\mathrm{z}}\right)=$

$\left(\mathrm{Ch}\right.$. Inducer efficiency / fungicide efficacy ( spray treatment $^{\mathrm{z}}$ )) X 100 
Data also indicated that, there was a relation between inducer material concentrations and their effect on purple blotch disease severity. Data clearly indicated that, increasing the concentration of inducer material led to increase their effect in reducing the disease severity (Tables $3 \& 4$ ). Data clearly showed the ability of some tested inducer materials to give more efficiency than tested fungicide (spray treatment) in reducing purple blotch disease (Tables $3 \& 4$ ). In this respect, IBA at $200 \mathrm{ppm}$ with dipping application was the best one in the growing season 2014-2015 (103.4\%) while, it gave $123.1 \%$ in the second season $2015-2016$.

Table 4. Effect of fungicide Amistar Top and some chemical inducers on onion purple blotch disease severity under field conditions during season 2015/2016

\begin{tabular}{|c|c|c|c|c|c|}
\hline \multicolumn{3}{|c|}{ Treatments } & \multicolumn{3}{|c|}{$2015-2016$} \\
\hline Dipping & Foliar & Conc. & $\begin{array}{l}\text { Disease } \\
\text { severity }\end{array}$ & Efficacy $^{\mathrm{x}}$ & $\begin{array}{l}\text { Fungicide } \\
\text { Efficacy }^{\mathrm{y}}\end{array}$ \\
\hline \multirow{11}{*}{ 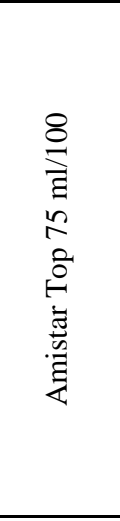 } & SA & $2 \mathrm{mM}$. & $10.22 \mathrm{ehgf}$ & 67.19 & 82.7 \\
\hline & SA & $4 \mathrm{mM}$. & $8.14 \mathrm{ihg}$ & 73.87 & 91.0 \\
\hline & SA & $8 \mathrm{mM}$. & $5.98 \mathrm{ij}$ & 80.80 & 99.5 \\
\hline & Bion & $2 \mathrm{mM}$. & $8.25 \mathrm{ihg}$ & 73.52 & 90.5 \\
\hline & Bion & $4 \mathrm{mM}$. & $6.02 \mathrm{ij}$ & 80.67 & 99.3 \\
\hline & Bion & $8 \mathrm{mM}$. & $4.12 \mathrm{kj}$ & 86.77 & 106.8 \\
\hline & IBA & $50 \mathrm{ppm}$ & $5.55 \mathrm{ij}$ & 82.18 & 101.2 \\
\hline & IBA & $100 \mathrm{ppm}^{1}$ & $2.14 \mathrm{kl}$ & 93.13 & 114.7 \\
\hline & IBA & $200 \mathrm{ppm}$ & 0.001 & 100.00 & 123.1 \\
\hline & Amistar Top & $300 \mathrm{ml} / \mathrm{fed}$. & 0.001 & 100.00 & 123.1 \\
\hline & \multicolumn{2}{|c|}{ Dipping only } & $27.25 \mathrm{~b}$ & 12.52 & 15.4 \\
\hline \multirow{11}{*}{ 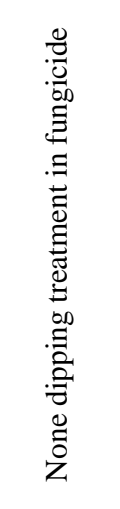 } & SA & $2 \mathrm{mM}$. & $16.45 \mathrm{c}$ & 47.19 & 58.1 \\
\hline & SA & $4 \mathrm{mM}$. & $12.75 \mathrm{ed}$ & 59.07 & 72.7 \\
\hline & SA & $8 \mathrm{mM}$. & $9.85 \mathrm{hgf}$ & 68.38 & 84.2 \\
\hline & Bion & $2 \mathrm{mM}$. & $14.05 \mathrm{~d}$ & 54.90 & 67.6 \\
\hline & Bion & $4 \mathrm{mM}$. & 10.8 egfd & 65.33 & 80.4 \\
\hline & Bion & $8 \mathrm{mM}$. & $8.15 \mathrm{ihg}$ & 73.84 & 90.9 \\
\hline & IBA & $50 \mathrm{ppm}$ & $12.3 \mathrm{edf}$ & 60.51 & 74.5 \\
\hline & IBA & $100 \mathrm{ppm}$ & $9.75 \mathrm{hgf}$ & 68.70 & 84.6 \\
\hline & IBA & $200 \mathrm{ppm}$ & $7.98 \mathrm{ih}$ & 74.38 & 91.6 \\
\hline & Amistar Top & $300 \mathrm{ml} / \mathrm{fed}$. & $5.85 \mathrm{ij}$ & $81.22^{z}$ & 100.0 \\
\hline & \multicolumn{2}{|c|}{ Control } & $31.15 \mathrm{a}$ & - & - \\
\hline
\end{tabular}

${ }^{\mathrm{x}} \%$ Treatment efficiency $=(($ Control-treatment $) /$ Control $) \mathrm{X} 100$

${ }^{\mathrm{y}} \%$ Chemical inducer efficiency to fungicide efficacy $\left(\right.$ spray treatment $\left.{ }^{\mathrm{z}}\right)=$ (Ch. Inducer efficiency / fungicide efficacy $\left(\right.$ spray treatment $\left.\left.^{\mathrm{z}}\right)\right)$ X 100 


\subsection{On onion bulb yield:}

Data presented in Table (5) indicate that all tested treatments caused significant increases in bulb yield during the two successive seasons 2014/2015 and 2015/2016 compared to untreated control.

Applying fungicide in dipping treatment with different concentrations of chemical inducers was significantly effective for increasing onion bulb yield if compared with without fungicide dipping treatment. Also, increasing concentration of chemical inducers gave more and significantly effective for increasing onion bulb yield.

The superior treatments in this regard were obtained by IBA at $200 \mathrm{ppm}$ with dipping fungicide application followed by bion at $8 \mathrm{mM}$ during two growing seasons 2014/2015 and 2015/2016 (Table 5).

Table 5. Effect of fungicide Amistar Top and some chemical inducers on onion bulb yield $\mathrm{kg} /$ plot under field conditions during seasons 2014/2015 and 2015/2016

\begin{tabular}{|c|c|c|c|c|c|c|}
\hline \multicolumn{3}{|c|}{ Treatments } & \multicolumn{2}{|c|}{$2014-2015$} & \multicolumn{2}{|c|}{$2015-2016$} \\
\hline Dipping & Foliar & Conc. & $\begin{array}{c}\text { Bulb } \\
\text { yield }^{\mathrm{y}}\end{array}$ & $\begin{array}{c}\text { Increases }^{\mathrm{Z}} \\
(\%)\end{array}$ & $\begin{array}{c}\text { Bulb } \\
\text { yield }^{\mathrm{y}}\end{array}$ & $\begin{array}{c}\text { Increases }^{\mathrm{Z}} \\
(\%)\end{array}$ \\
\hline \multirow{11}{*}{ 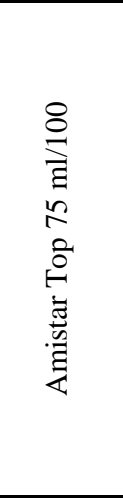 } & $\mathrm{SA}$ & $2 \mathrm{mM}$. & 25.5 fge & 64.52 & 28.911 & 55.38 \\
\hline & $\mathrm{SA}$ & $4 \mathrm{mM}$. & $31.2 \mathrm{bdec}$ & 101.29 & 35.2 gifh & 89.25 \\
\hline & $\mathrm{SA}$ & $8 \mathrm{mM}$. & $33.3 \mathrm{bdec}$ & 114.84 & 37.5 gdfce & 101.61 \\
\hline & Bion & $2 \mathrm{mM}$. & 30.2 fbdec & 94.84 & 34.1 gihj & 83.33 \\
\hline & Bion & $4 \mathrm{mM}$ & $32.4 \mathrm{bdec}$ & 109.03 & 36.5 gifhe & 96.24 \\
\hline & Bion & $8 \mathrm{mM}$ & 34.8 bdac & 124.52 & 39.2 dce & 110.75 \\
\hline & IBA & $50 \mathrm{ppm}$ & $33.1 \mathrm{bdec}$ & 113.55 & 37.3 gdfhe & 100.54 \\
\hline & IBA & $100 \mathrm{ppm}$ & $36.1 \mathrm{bac}$ & 132.90 & $40.6 \mathrm{dc}$ & 118.28 \\
\hline & IBA & $200 \mathrm{ppm}$ & $39.3 \mathrm{ba}$ & 153.55 & $44.2 \mathrm{~b}$ & 137.63 \\
\hline & $\begin{array}{c}\text { Amistar } \\
\text { Top }\end{array}$ & $\begin{array}{c}300 \\
\mathrm{ml} / \text { fed }\end{array}$ & $43.4 \mathrm{a}$ & 180.00 & $48.8 \mathrm{a}$ & 162.37 \\
\hline & \multicolumn{2}{|c|}{ Dipping only } & $20.3 \mathrm{gh}$ & 31.29 & $30.97 \mathrm{lkj}$ & 33.87 \\
\hline \multirow{11}{*}{ 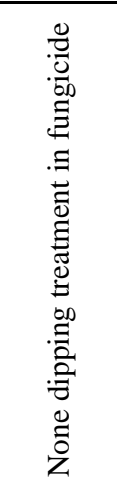 } & $\mathrm{SA}$ & $2 \mathrm{mM}$. & $21.4 \mathrm{fgh}$ & 38.06 & $24.3 \mathrm{~m}$ & 30.65 \\
\hline & $\mathrm{SA}$ & $4 \mathrm{mM}$. & 27.9 fgdec & 80.00 & $31.5 \mathrm{lkj}$ & 69.35 \\
\hline & $\mathrm{SA}$ & $8 \mathrm{mM}$. & 30.4 bdec & 96.13 & 34.3 gihj & 84.41 \\
\hline & Bion & $2 \mathrm{mM}$. & 26.8 fgde & 72.90 & $30.3 \mathrm{lk}$ & 62.90 \\
\hline & Bion & $4 \mathrm{mM}$. & $29.3 \mathrm{fdec}$ & 89.03 & $33.1 \mathrm{ikj}$ & 77.96 \\
\hline & Bion & $8 \mathrm{mM}$. & $32.1 \mathrm{bdec}$ & 107.10 & 36.2 gifhe & 94.62 \\
\hline & IBA & $50 \mathrm{ppm}$ & $30.1 \mathrm{fbdec}$ & 94.19 & $34.0 \mathrm{ihj}$ & 82.80 \\
\hline & IBA & $100 \mathrm{ppm}$ & $33.5 \mathrm{bdec}$ & 116.13 & 37.8 dfce & 103.23 \\
\hline & IBA & $200 \mathrm{ppm}$ & $36.2 \mathrm{bac}$ & 133.55 & $40.8 \mathrm{c}$ & 119.35 \\
\hline & $\begin{array}{c}\text { Amistar } \\
\text { Top }\end{array}$ & $\begin{array}{c}300 \\
\mathrm{ml} / \text { fed }\end{array}$ & 39.0 ba & 151.61 & $43.9 \mathrm{~b}$ & 136.02 \\
\hline & \multicolumn{2}{|c|}{ Control } & $15.5 \mathrm{~h}$ & - & $18.6 \mathrm{n}$ & - \\
\hline
\end{tabular}

${ }^{\mathrm{y}}$ Onion bulb yield kg/plot $(10.5 \mathrm{~m} 2=1 / 400$ fed.)

${ }^{\mathrm{z}}$ Increases related to the control 
2. Effect of foliar spraying with some chemical inducers, applied with or without fungicide treatment on biochemical changes in onion plants:

2.1. On phenolic contents in onion leaves:

Results presented in Tables (6\&7) indicate that, phenol contents including the free, conjugated and total phenols were obviously higher in leaves of onion plants which treated with any tested chemical inducers than at untreated control during the two growing seasons 2014/2015 and 2015/2016.

The highest phenolic contents were induced by IBA treatment at $200 \mathrm{ppm}$ and SA treatment at $8 \mathrm{mM}$. Data clearly indicated that, phenol contents were affected by three tested concentrations of each chemical inducer. But, applying fungicide as dipping treatment with different concentrations of chemical inducers had no significant effect in increasing phenolic contents (free, conjugated and total phenols) in leaves of onion plants during the two growing seasons 2014-2015 and 2015-2016.

Table 6. Effect of fungicide Amstar Top and some chemical inducers on phenol contents (milligrams equivalent of catechol /g) in onion leaves during season 2014/2015

\begin{tabular}{|c|c|c|c|c|c|}
\hline \multicolumn{3}{|c|}{ Treatments } & \multicolumn{3}{|c|}{ 2014-2015 } \\
\hline Dipping & Foliar & Conc. & Free & Conjugate & Total \\
\hline \multirow{11}{*}{ 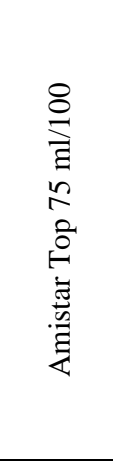 } & SA & $2 \mathrm{mM}$. & $11.69 \mathrm{~g}$ & $5.75 \mathrm{egf}$ & $17.44 \mathrm{i}$ \\
\hline & SA & $4 \mathrm{mM}$. & $14.75 \mathrm{fde}$ & 5.9 egf & $20.65 \mathrm{~h}$ \\
\hline & SA & $8 \mathrm{mM}$. & $16.4 \mathrm{bdec}$ & $6.66 \mathrm{edc}$ & $23.06 \mathrm{dfe}$ \\
\hline & Bion & $2 \mathrm{mM}$. & $14.94 \mathrm{fde}$ & $6.33 \mathrm{edf}$ & $21.27 \mathrm{gh}$ \\
\hline & Bion & $4 \mathrm{mM}$. & $15.63 \mathrm{fdec}$ & $7.37 \mathrm{bac}$ & $23.00 \mathrm{dfe}$ \\
\hline & Bion & $8 \mathrm{mM}$. & $16.19 \mathrm{fbdec}$ & $7.45 \mathrm{bac}$ & $23.64 \mathrm{dfe}$ \\
\hline & IBA & $50 \mathrm{ppm}$ & $16.25 \mathrm{fbdec}$ & $7.66 \mathrm{ba}$ & $23.91 \mathrm{dce}$ \\
\hline & IBA & $100 \mathrm{ppm}$ & $17.31 \mathrm{bac}$ & $7.82 \mathrm{ba}$ & $25.13 \mathrm{bc}$ \\
\hline & IBA & $200 \mathrm{ppm}$ & $18.38 \mathrm{a}$ & $8.15 \mathrm{a}$ & $26.53 \mathrm{a}$ \\
\hline & Amistar Top & $300 \mathrm{ml} / \mathrm{fed}$ & $11.44 \mathrm{~g}$ & $5.38 \mathrm{gf}$ & $16.82 \mathrm{i}$ \\
\hline & \multicolumn{2}{|c|}{ Dipping only } & $9.31 \mathrm{hi}$ & $2.77 \mathrm{~h}$ & $12.08 \mathrm{j}$ \\
\hline \multirow{11}{*}{ 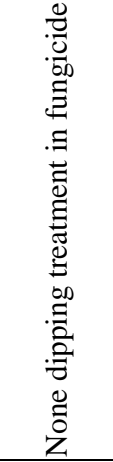 } & SA & $2 \mathrm{mM}$. & $11.25 \mathrm{~g}$ & $5.43 \mathrm{gf}$ & $16.89 \mathrm{i}$ \\
\hline & SA & $4 \mathrm{mM}$. & $14.22 \mathrm{f}$ & $5.54 \mathrm{gf}$ & $20.01 \mathrm{~h}$ \\
\hline & SA & $8 \mathrm{mM}$. & $15.81 \mathrm{fbdec}$ & $6.26 \mathrm{edf}$ & $22.34 \mathrm{gf}$ \\
\hline & Bion & $2 \mathrm{mM}$. & $14.44 \mathrm{fe}$ & $6.00 \mathrm{egf}$ & $20.64 \mathrm{~h}$ \\
\hline & Bion & $4 \mathrm{mM}$. & $15.08 \mathrm{fde}$ & $6.99 \mathrm{bdc}$ & $22.31 \mathrm{gf}$ \\
\hline & Bion & $8 \mathrm{mM}$. & $15.60 \mathrm{fdec}$ & $7.03 \mathrm{bdc}$ & $22.91 \mathrm{fe}$ \\
\hline & IBA & $50 \mathrm{ppm}$ & $15.72 \mathrm{fbdec}$ & $7.30 \mathrm{bac}$ & $23.23 \mathrm{dfe}$ \\
\hline & IBA & $100 \mathrm{ppm}$ & $16.73 \mathrm{bdac}$ & $7.43 \mathrm{bac}$ & $24.40 \mathrm{dc}$ \\
\hline & IBA & $200 \mathrm{ppm}$ & $17.75 \mathrm{ba}$ & 7.72 ba & $25.74 \mathrm{ba}$ \\
\hline & Amistar Top & $300 \mathrm{ml} / \mathrm{fed}$ & $11.01 \mathrm{hg}$ & $5.06 \mathrm{~g}$ & $16.28 \mathrm{i}$ \\
\hline & \multicolumn{2}{|c|}{ Control } & $8.89 \mathrm{i}$ & $2.48 \mathrm{~h}$ & $11.60 \mathrm{j}$ \\
\hline
\end{tabular}


Table 7. Effect of fungicide Amistar Top and some chemical inducers on phenol contents (milligrams equivalent of catechol $/ g$ ) in onion leaves during season 2015/2016

\begin{tabular}{|c|c|c|c|c|c|}
\hline \multicolumn{3}{|c|}{ Treatments } & \multicolumn{3}{|c|}{$2015-2016$} \\
\hline Dipping & Foliar & Conc. & Free & Conjugate & Total \\
\hline \multirow{11}{*}{ 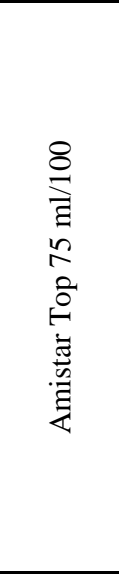 } & SA & $2 \mathrm{mM}$. & $11.44 \mathrm{i}$ & $5.68 \mathrm{ghf}$ & $17.13 \mathrm{gh}$ \\
\hline & SA & $4 \mathrm{mM}$. & $14.44 \mathrm{gh}$ & $5.83 \mathrm{e}$ ghf & 20.27 ef \\
\hline & SA & $8 \mathrm{mM}$. & $16.06 \mathrm{ed}$ & 6.58 edc & $22.64 \mathrm{ebdc}$ \\
\hline & Bion & $2 \mathrm{mM}$. & $14.63 \mathrm{ghf}$ & $6.25 \mathrm{edf}$ & 20.88 edfef \\
\hline & Bion & $4 \mathrm{mM}$. & 15.30 gef & $7.28 \mathrm{bac}$ & $22.58 \mathrm{ebdc}$ \\
\hline & Bion & $8 \mathrm{mM}$. & $15.85 \mathrm{de}$ & $7.36 \mathrm{abc}$ & 23.21 ebdac \\
\hline & IBA & $50 \mathrm{ppm}$ & $15.91 \mathrm{fbde}$ & $7.56 \mathrm{ba}$ & $23.47 \mathrm{bdac}$ \\
\hline & IBA & $100 \mathrm{ppm}$ & $16.95 \mathrm{bac}$ & 7.72 ba & $24.68 \mathrm{bac}$ \\
\hline & IBA & 200 ppm & $17.99 \mathrm{a}$ & $8.05 \mathrm{a}$ & $26.04 \mathrm{a}$ \\
\hline & Amistar Top & $300 \mathrm{ml} / \mathrm{fed}$ & $11.20 \mathrm{i}$ & $5.31 \mathrm{~h}$ & $16.51 \mathrm{~h}$ \\
\hline & \multicolumn{2}{|c|}{ Dipping only } & $9.12 \mathrm{j}$ & $2.74 \mathrm{i}$ & $11.86 \mathrm{i}$ \\
\hline \multirow{11}{*}{ 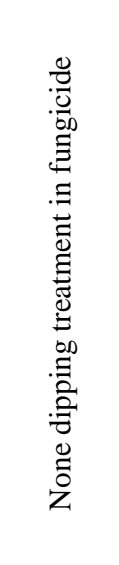 } & SA & $2 \mathrm{mM}$. & $11.01 \mathrm{i}$ & $5.36 \mathrm{gh}$ & $16.58 \mathrm{~h}$ \\
\hline & SA & $4 \mathrm{mM}$. & $13.92 \mathrm{~h}$ & $5.48 \mathrm{ghf}$ & $19.63 \mathrm{gf}$ \\
\hline & SA & $8 \mathrm{mM}$. & $15.48 \mathrm{def}$ & 6.18 egdf & 21.93 edfc \\
\hline & Bion & $2 \mathrm{mM}$. & $14.13 \mathrm{~h}$ & $5.92 \mathrm{egf}$ & $20.26 \mathrm{ef}$ \\
\hline & Bion & $4 \mathrm{mM}$. & $14.76 \mathrm{ghf}$ & $6.90 \mathrm{bdc}$ & 21.90 edfc \\
\hline & Bion & $8 \mathrm{mM}$. & 15.27 gef & $6.95 \mathrm{bdc}$ & 22.48 ebdfc \\
\hline & IBA & $50 \mathrm{ppm}$ & 15.39 gef & $7.20 \mathrm{bac}$ & $22.80 \mathrm{ebdc}$ \\
\hline & IBA & $100 \mathrm{ppm}$ & $16.38 \mathrm{dc}$ & $7.33 \mathrm{bac}$ & $23.96 \mathrm{bac}$ \\
\hline & IBA & 200 ppm & $17.37 \mathrm{ba}$ & $7.62 \mathrm{ba}$ & $25.26 \mathrm{ba}$ \\
\hline & Amistar Top & $300 \mathrm{ml} / \mathrm{fed}$ & $10.77 \mathrm{i}$ & $5.00 \mathrm{~h}$ & $15.98 \mathrm{~h}$ \\
\hline & \multicolumn{2}{|c|}{ Control } & $8.70 \mathrm{j}$ & $2.45 \mathrm{i}$ & $11.39 \mathrm{i}$ \\
\hline
\end{tabular}

\subsection{On some oxidative reductive enzymes in onion leaves:}

Results in Tables (8\&9) show that, catalase (CAT), peroxidase (PO) and polyphenoloxidase (PPO) activities in onion leaves increased as result of using any chemical inducer treatments than the untreated control during two growing seasons 2014/2015 and 2015/2016.

In general, applying fungicide as dipping treatment with different concentrations of chemical inducers had no significant effect for increasing enzymes activities in leaves of onion plants. Data clearly indicated that, enzymes activities were affected by three tested concentrations of each chemical inducer. In this respect, the highest activities in all determine enzymes were induced by IBA at $200 \mathrm{ppm}$ and SA at 8 $\mathrm{mM}$ during the two growing seasons 2014-2015 and 2015-2016. 
Table 8. Effect of fungicide Amistar Top and some chemical inducers on enzymes activities in onion leaves during seasons 2014/2015

\begin{tabular}{|c|c|c|c|c|c|}
\hline \multicolumn{3}{|c|}{ Seasons } & \multicolumn{3}{|c|}{ 2014-2015 } \\
\hline Dipping & Foliar & Conc. & $\mathrm{PO}$ & PPO & CAT \\
\hline \multirow{11}{*}{ 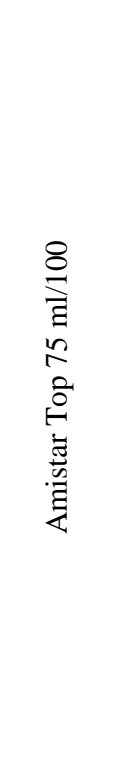 } & SA & $2 \mathrm{mM}$. & $1.169 \mathrm{f}$ & $0.482 \mathrm{f}$ & $0.327 \mathrm{j}$ \\
\hline & SA & $4 \mathrm{mM}$. & $1.475 \mathrm{e}$ & $0.608 \mathrm{ed}$ & $0.413 \mathrm{hi}$ \\
\hline & SA & $8 \mathrm{mM}$. & 1.640 ebdac & 0.676 ebdac & 0.459 dce \\
\hline & Bion & $2 \mathrm{mM}$. & $1.494 \mathrm{ed}$ & $0.616 \mathrm{ed}$ & 0.418 ghi \\
\hline & Bion & $4 \mathrm{mM}$. & $1.563 \mathrm{edc}$ & 0.644 edc & 0.437 ghfe \\
\hline & Bion & $8 \mathrm{mM}$. & $1.619 \mathrm{ebdc}$ & $0.667 \mathrm{ebdc}$ & $0.453 \mathrm{dfe}$ \\
\hline & IBA & $50 \mathrm{ppm}$ & $1.625 \mathrm{ebdc}$ & 0.670 ebdac & $0.455 \mathrm{dfe}$ \\
\hline & IBA & 100 ppm & $1.731 \mathrm{bac}$ & $0.714 \mathrm{bac}$ & $0.484 \mathrm{bc}$ \\
\hline & IBA & $200 \mathrm{ppm}$ & $1.838 \mathrm{a}$ & $0.758 \mathrm{a}$ & $0.514 \mathrm{a}$ \\
\hline & Amistar Top & $300 \mathrm{ml} / \mathrm{fed}$ & $1.144 \mathrm{f}$ & $0.472 \mathrm{f}$ & $0.320 \mathrm{j}$ \\
\hline & \multicolumn{2}{|c|}{ Dipping only } & $0.931 \mathrm{~g}$ & $0.384 \mathrm{gh}$ & $0.261 \mathrm{k}$ \\
\hline \multirow{11}{*}{ 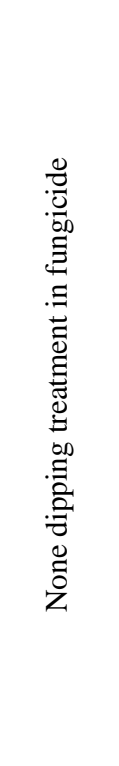 } & SA & $2 \mathrm{mM}$. & $1.146 \mathrm{f}$ & $0.473 \mathrm{f}$ & $0.321 \mathrm{j}$ \\
\hline & SA & $4 \mathrm{mM}$. & $1.446 \mathrm{e}$ & $0.596 \mathrm{e}$ & $0.405 \mathrm{i}$ \\
\hline & SA & $8 \mathrm{mM}$. & $1.608 \mathrm{ebdc}$ & $0.663 \mathrm{ebdc}$ & $0.450 \mathrm{dfe}$ \\
\hline & Bion & $2 \mathrm{mM}$. & $1.465 \mathrm{e}$ & $0.604 \mathrm{e}$ & $0.410 \mathrm{i}$ \\
\hline & Bion & $4 \mathrm{mM}$. & $1.532 \mathrm{edc}$ & $0.631 \mathrm{edc}$ & 0.428 ghfi \\
\hline & Bion & $8 \mathrm{mM}$. & $1.587 \mathrm{edc}$ & $0.654 \mathrm{ebdc}$ & 0.444 gfe \\
\hline & IBA & $50 \mathrm{ppm}$ & $1.593 \mathrm{edc}$ & $0.657 \mathrm{ebdc}$ & $0.446 \mathrm{fe}$ \\
\hline & IBA & 100 ppm & $1.697 \mathrm{bdac}$ & $0.700 \mathrm{bdac}$ & $0.475 \mathrm{dc}$ \\
\hline & IBA & $200 \mathrm{ppm}$ & $1.802 \mathrm{ba}$ & $0.743 \mathrm{ba}$ & $0.504 \mathrm{ba}$ \\
\hline & Amistar Top & $300 \mathrm{ml} / \mathrm{fed}$ & $1.122 \mathrm{f}$ & $0.463 \mathrm{f}$ & $0.314 \mathrm{j}$ \\
\hline & \multicolumn{2}{|c|}{ Control } & $0.913 \mathrm{~g}$ & $0.376 \mathrm{~h}$ & $0.256 \mathrm{k}$ \\
\hline
\end{tabular}


Table 9. Effect of fungicide Amistar Top and some chemical inducers on enzymes activities in onion leaves during season 2015/2016

\begin{tabular}{|c|c|c|c|c|c|}
\hline \multicolumn{3}{|c|}{ Seasons } & \multicolumn{3}{|c|}{$2015-2016$} \\
\hline Dipping & Foliar & Conc. & $\mathrm{PO}$ & PPO & CAT \\
\hline \multirow{11}{*}{ 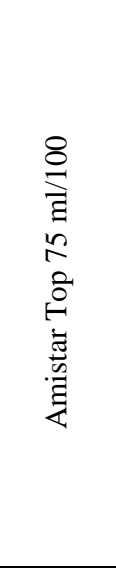 } & SA & $2 \mathrm{mM}$. & $1.144 \mathrm{e}$ & $0.472 \mathrm{e}$ & $0.320 \mathrm{e}$ \\
\hline & SA & $4 \mathrm{mM}$. & $1.463 \mathrm{~cd}$ & $0.603 \mathrm{~d}$ & $0.409 \mathrm{dc}$ \\
\hline & SA & $8 \mathrm{mM}$. & $1.573 \mathrm{~b}$ & 0.649 bdac & 0.440 bdac \\
\hline & Bion & $2 \mathrm{mM}$. & $1.494 \mathrm{cbd}$ & $0.616 \mathrm{dc}$ & $0.418 \mathrm{dc}$ \\
\hline & Bion & $4 \mathrm{mM}$. & $1.530 \mathrm{cbd}$ & $0.631 \mathrm{bdc}$ & $0.428 \mathrm{bdc}$ \\
\hline & Bion & $8 \mathrm{mM}$. & $1.559 \mathrm{cb}$ & 0.643 bdac & 0.436 bdac \\
\hline & IBA & $50 \mathrm{ppm}$ & $1.578 \mathrm{~b}$ & 0.651 bdac & 0.441 bdac \\
\hline & IBA & 100 ppm & $1.702 \mathrm{a}$ & $0.702 \mathrm{ba}$ & $0.476 \mathrm{ba}$ \\
\hline & IBA & $200 \mathrm{ppm}$ & $1.741 \mathrm{a}$ & $0.718 \mathrm{a}$ & $0.487 \mathrm{a}$ \\
\hline & Amistar Top & $300 \mathrm{ml} / \mathrm{fed}$ & $1.129 \mathrm{e}$ & $0.466 \mathrm{e}$ & $0.316 \mathrm{e}$ \\
\hline & \multicolumn{2}{|c|}{ Dipping only } & $0.882 \mathrm{f}$ & $0.364 \mathrm{f}$ & $0.247 \mathrm{f}$ \\
\hline \multirow{11}{*}{ 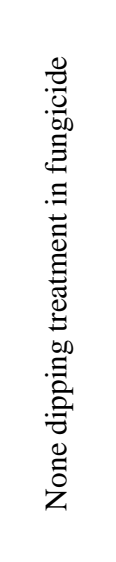 } & SA & $2 \mathrm{mM}$. & $1.122 \mathrm{e}$ & $0.463 \mathrm{e}$ & $0.314 \mathrm{e}$ \\
\hline & SA & $4 \mathrm{mM}$. & $1.434 \mathrm{~d}$ & $0.591 \mathrm{~d}$ & $0.401 \mathrm{~d}$ \\
\hline & SA & $8 \mathrm{mM}$. & $1.542 \mathrm{cb}$ & $0.636 \mathrm{bdac}$ & 0.431 bdac \\
\hline & Bion & $2 \mathrm{mM}$. & $1.465 \mathrm{~cd}$ & $0.604 \mathrm{~d}$ & $0.410 \mathrm{dc}$ \\
\hline & Bion & $4 \mathrm{mM}$. & $1.500 \mathrm{cbd}$ & $0.619 \mathrm{~d}$ & $0.420 \mathrm{bdc}$ \\
\hline & Bion & $8 \mathrm{mM}$. & $1.528 \mathrm{cbd}$ & $0.630 \mathrm{bdac}$ & $0.427 \mathrm{bdc}$ \\
\hline & IBA & $50 \mathrm{ppm}$ & $1.547 \mathrm{cb}$ & 0.638 bdac & 0.432 bdac \\
\hline & IBA & 100 ppm & $1.669 \mathrm{a}$ & $0.688 \mathrm{bac}$ & $0.467 \mathrm{bac}$ \\
\hline & IBA & $200 \mathrm{ppm}$ & $1.707 \mathrm{a}$ & 0.704 ba & $0.477 \mathrm{ba}$ \\
\hline & Amistar Top & $300 \mathrm{ml} / \mathrm{fed}$ & $1.107 \mathrm{e}$ & $0.457 \mathrm{e}$ & $0.310 \mathrm{e}$ \\
\hline & \multicolumn{2}{|c|}{ Control } & $0.865 \mathrm{f}$ & $0.357 \mathrm{f}$ & $0.242 \mathrm{f}$ \\
\hline
\end{tabular}

\section{D i s c u s s i on}

Regarding to the effect of treatments by chemical inducers on onion diseases severity of downy mildew and purple blotch, results showed that, there was a significant effect of all treatments tested at different concentrations in reducing studied diseases and consequently increasing the bulb yield during two successive seasons 2014/2015 and 2015/2016 compared to untreated control. This agrees with many investigators who stated that, the inducer resistance has an important role in controlling of plant diseases (Mandal et al., 2009; Hussien 2011; Ibrahim et al., 2013 and Mahmoud et al., 2014). IBA and bion treatments showed appreciable increases in the phenolic contents and increased the activity of catalase (CAT), peroxidase (PO) and polyphenoloxidase (PPO) in onion leaves that led to reduce the 
severity of onion downy mildew and purple blotch diseases by enhancing activities of the defense related enzymes moreover it increased phenolic contents including the free, conjugated and total phenols. This is in agreement with Khalifa, 2003, Chowdhury (2003), Mahmoud et al. (2011) and Ragab et al. (2012) who stated that, IBA showed increase in the total phenol, calcium and increase the activity of many enzymes included peroxidase, polyphenoloxidase, catalase and pectolytic enzyme. While, bion has the ability to enhance the activities of the defense related enzymes chitinase, B-(1, 3)-gluconase and peroxidase (Siegrist et al., 1997; Abou-Taleb, 2001; Mosa 2002 and Mahmoud et al., 2014). Moreover, in many plants investigated so far, bion treatment is associated with increases in activities of many classes of pathogenesis-related protein (Gorlach et al., 1996; Schweizer et al., 1999 and AbouTaleb, 2001).

The results of this study indicated that recommended fungicide Amistar Top was the best treatment for reducing disease severity of downy mildew and purple blotch under field conditions during the two growing seasons (2014-2015 and 2015-2016) compared to untreated control treatment. This is in accordance with Survilienè et al. (2008) and Mahmoud et al. (2013) who stated that Amistar SC 25\% and Amistar Top SC $32.5 \%$ gave significant suppression in disease severity of onion downy mildew and purple blotch and increased the marketable onion yield. Moreover, some reports suggested that fungicides containing active ingredients such as azoxystrobin, benalaxyl, dimethomorf, metalaxyl and Difenoconazole are the most effective resulted in control of onion downy mildew (Robak and Adamicki, 2007 and Mahmoud et al., 2013).

In the present study, using fungicide Amistar Top as dipping treatment in combinations with different concentrations of chemical inducers used as spraying treatment were significantly effective for reducing diseases severity of downy mildew and purple blotch diseases under naturally conditions in field trials compared with untreated control (without any treatment). This is in agreement with Spencer et al. (2003) whom reported that, treated the transplants for many crops i.e. brassicas, soybean, peas and sunflower with fungicide led to more control of downy mildew diseases. That might be due to the infection with downy mildew fungi happens by two ways: first way onion transplant infected by the mycelium or oospores which left on plant remains in the field after harvesting (systemic infected) and the second way the plant leaf infected by sporangium spores (Local infection). In this respect, the first ways affected by using application Amistar Top as dipping treatment and give more effect for controlling downy mildew (Spencer et al., 2003).

\section{References}

Abou-Taleb, M.A. 2001. Biochemical changes associated with the application of some resistance-inducing compounds for controlling powdery mildew of cucumber. Egypt. J. Appl. Sci., 16: 387-405.

Akter, U.S.; Rashid, H.; Rahman, A.; Islam, R. and Haque M. 2015. Effect of the treatments in controlling purple blotch complex of onion (Allium cepa L.). Academic J. Plant Sci., 7(2): 14-19. 
Allam, A.I. and Hollis S.P. 1972. Sulfide inhibition of oxidase in rice root. Phytopathology, 62: 634-639.

Chowdhury, A.K. 2003. Control of Sclerotium blight of groundnut by plant growth substances. Crop Res. (Hisar) 25: $355-359$.

Conn, K.L. and Tewari J.P. 1990. Survey of Alternaria black spot and Sclerotinia stem rot in central Alberta in 1989. Can. Plant Dis. Survey, 70: 66-67.

Gaikwad K.N.; Jadhav, Shobha, U. and Kakulte, V.R. 2014. Management of fungal diseases of onion (Allium cepa L) by using plant extraction. Life Sci. Botany, 4(2): 28-30.

Gorlach, J.; Volorath, S.; Knaut-Beiter, G.; Hengy, G.; Beckhove, U.; Kogel, K.H.; Ostendrop, M.; Staub, T.; Ward, E.; Kessmann, H. and Ryals, J. 1996. Benzothiadiazol a novel class of inducers of systematic acquired resistance activate gene expression and disease resistance in wheat. Plant Cell, 8: 629-643.

Gupta R.C.; Yadav, S.P. and Srivastava, K.J. 2011. Studies on management of foliar diseases of onion (Allium cepa L.). through recommended pesticides and their residue status after harvest. In. Nat. Symp. on Alliums: Current Scenario and Emerging Trends, 12-14 March 2011, Pune, pp. 234-35.

Hussien, Zeinab N. 2011. New approaches for controlling peanut root rot and pod rots caused by Rhizoctonia solani in Egypt and Nigeria. Ph.D. Thesis. African Research and Studies Inst., Cairo Univ., 138 p.

Ibrahim, M.M.; Khalifa, M.M.A. and Mahmoud, E.Y. 2013. Using of some chemical inducers on controlling peanut Cercospora leaf spot as one of the possible alternative to fungicides. Egypt J. Appl. Sci., 28(7): 31-46.

Khalifa, M.M.A. 2003. Pathological studies on charcoal rot disease of sesame. Ph.D. Thesis, Fac. of Agric. Moshtohor, Zagazig Univ. 273 p.

Mahmoud, E.Y.; Ata, A.A. and Mohamed, H.A. 2011. Efficiency of some growth regulators as inducer resistance factor for controlling peanut damping-off, wilt and root rots diseases. Fayoum J. Agric. Res. Dev., 25(2):152-163.

Mahmoud, E.Y.; Saleh Wagida, A.M. and Hussien Zeinab, N. 2014. Biochemical change associated with induced resistance to peanut root and pod rots diseases. Minufiya J. Agric. Res., 39, 4(1): 1227-1253.

Mahmoud, E.Y.; M.M. Ibrahim, Mostafa Fatma, A. and Hussien Zeinab N. 2016. Integration of Bacillus subtilis and with some essential plant oils for the control of onion white rot. Egypt. J. Agric. Res., 94(3): 591-608.

Mahmoud, Noher A.; Khalifa, M.M.A. and Abou-Zeid, N.M. 2013. Performance of some biofungicides on the most onion economic diseases compared to recommended fungicide in Egypt. II- Downy mildew and purple blotch diseases control and their economical feasibility. Egypt. J. Appl. Sci., 28(1): 66-92. 
Mandal, S.; Mallicka, N. and Mitraa, A. 2009. Salicylic acid-induced resistance to Fusarium oxysporum f. sp. lycopersici in tomato. Plant Physiol. Bioch., 47(7): 642-649.

Mathur, K. and Sharma, S.N. 2006. Evaluation of fungicides against Alternaria porri and Stemphylium vesicarium disease of onion in Rajasthan. J. Mycol. PIant Pathol., 36(2):88-97.

Matta, A. and Dimond A.E. 1963. Symptoms of Fusarium wilt in relation to quantity of fungus and enzyme activity in tomato stems. Phytopathology, 53: 547-587.

Maude, R.B. 1990. "Leaf Diseases of Onions". In: Onion and allied crops, (H.D. Rabinowitch \& J.L. Brewster Eds.), CRC Press, Inc. Boca Raton Florida, USA, pp.173-212.

Maxwell, D.P. and D.F. Bateman, 1967. Changes in the activities of some oxidases in extracts of Rhizoctonia infected bean hypocotyle in relation to lesion maturation. Phytopathology, 57: 132-136.

Meena, B.; Marimuthu, T. and Velazhahan, R. 2001. Salicylic acid induces systemic resistance in groundnut against late leaf spot caused by Cercosporidium personatum. J. Mycol. Plant Path., 31:139-145. (C.F. CAB Abstracts 2003).

Mosa, A.A. 2002. Induced resistance in rice against blast disease using abiotic and biotic agents. Annales Agric. Sci., Ain Shams Univ., Cairo, Egypt, 47: 993-1008.

MSTAT-C. 1991. A Software Program for the Design, Management and Analysis of Agronomic Research Experiments. Michigan State University, pp: 400.

Mustafijur, R.; Sikder, M.; Shohana, N. and Abul, K. 2015. In vitro evaluation of botanical extract, bioagents and fungicides against purple blotch disease of bunch onion in Bangladesh. Advances Zool. Botany, 3(4): 179-183.

Ragab Seham, S.; Naffa Azza, M. and Mahmoud, E.Y. 2012. Induced resistance for controlling Tomato fruits rots by using safe chemicals. Egypt J. Phytopathol., 40:29-41.

Robak, J. and Adamicki, F. 2007. The effect of pre-harvest treatment with fungicide on the storage potential of root vegetables. Vegetable Crops Res., Bulletin, 67: 187-196.

Rondomanski, W. 1971. The sources of primary infection of onion downy mildew (Peronospora destructor (Berk.) Fries). Tagunsbericht Deutsche Akademie der Landwirtschaftswissenschaften zu Berlin, 115: 157-171.

Schwartz, H.F. and Mohan, S.K. 2008. Compendium of onion and garlic diseases. St. Paul: APS. 127p.

Schweizer, P.; Schlagenhauf, E.; Schaffrath, U. and Dudler, R. 1999. Different patterns of host genes are induced in rice by Pseudomonas syringae a biological inducer of resistance, and the chemical inducer benzothiadiazol (BTH). Eur. J. Plant Pathol., 105: 659-665. 
Sharma, R.C.; Gill, S.S. and Kohli, N. 2002. Pathological problems in production and storage of onion seed in Punjab and their remedial measures. Seed Research, 30, 134-141.

Siegrist, J.; Glenewinkle, D.; Kolle, C. and Schmidtke, M. 1997. Chemically induced resistance in green bean against bacterial and fungal pathogens. Plant Dis., 104: 599-610.

Snell, F.D. and Snell, C.I. 1953." Colorimetric Methods". Vol. III. D. Van Nostrand Co. Inc., Torento, N. Y., London, 606 p.

Spencer, P.T.N.; Gisi, U.; Bristol, U.K. and Lebeda, A. 2003. Advances in Downy Mildew Research. Kluwer Acadamic Publishers, New York, Boston, Dordrecht, London, Moscow 284p.

Survilienè, E.; Valiuškaite, A. and Raudonis, L. 2008. The effect of fungicides on the development of downy mildew of onions. Zemdirbyste Agriculture, 95(3): 171-179.

Tomaz, I.L. and Lima, A. 1988. An important disease of onion caused by Stemphylium vesicarium (Wallr.) Simmons in Portugal, Hortic. Abst., 58:618.

Townsend, G.R. and Heuberger J.W. 1943. Methods for estimating losses caused by disease in fungicide experiments. Plt Dis. Reptr., 27(17): 340-343.

Tripathy, P.; Patel, D.; Sahoo, B.B.; Das, S.K. and Dash, D.K. 2014. Studies on management of foliar diseases in onion (Allium cepa L.). J. Crop Weed, 10(2):457-460.

Walters, D.; Newton, A. and Lyon, G. 2007. Induced Resistance for Plant Defence. Blackwell Publishing Editorial Offices, 269 p.

(Received 20/08/2016; in revised form 15/10/2016) 


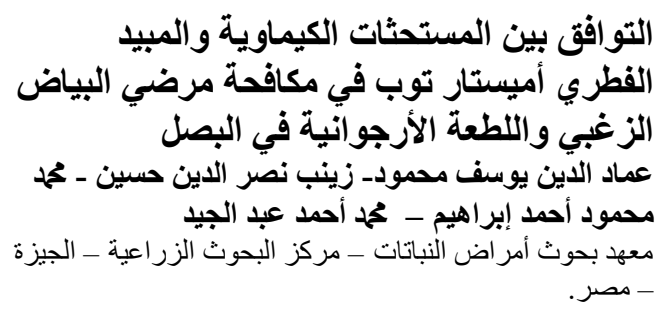

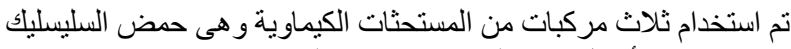

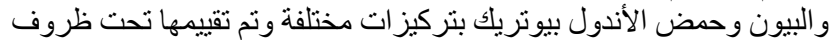

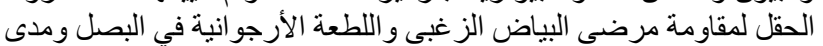

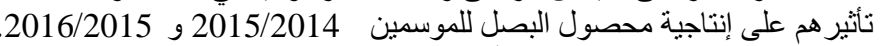

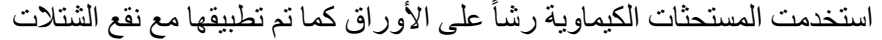

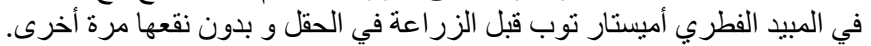

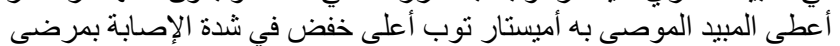

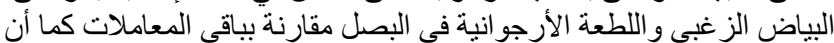

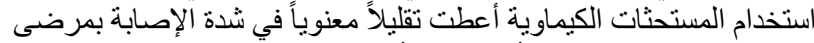

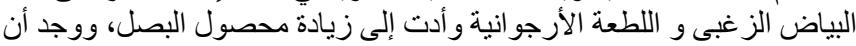

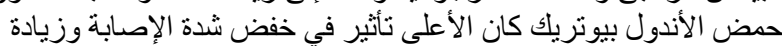

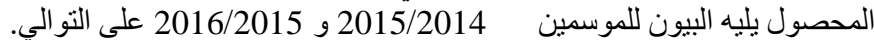

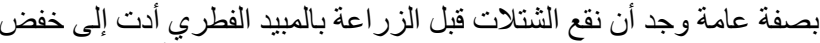

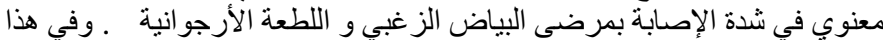

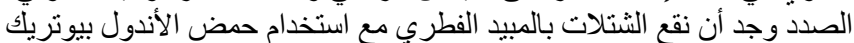

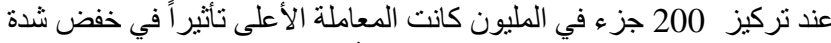

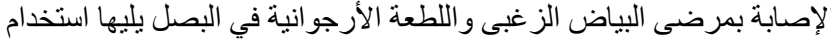

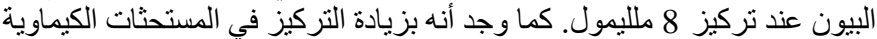

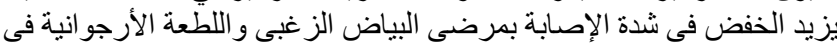

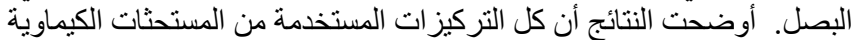

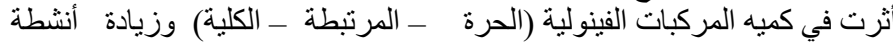

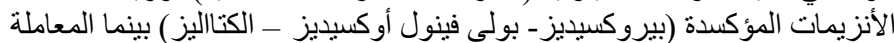

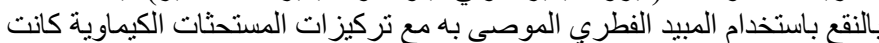

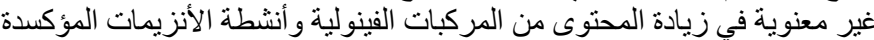

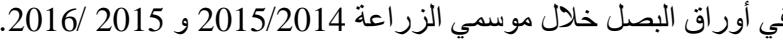

Egypt. J. Phytopathol., Vol. 44, No. 2 (2016) 